\title{
Towards Billion-Body Dynamics Simulation of Granular Material
}

\author{
Rebecca Shotwell Dan Negrut \\ Department of Mechanical Engineering \\ University of Wisconsin - Madison
}

\begin{abstract}
Advances are being made in the simulation of granular material, such that billionbody simulations are, perhaps, feasible using a combination of existing hardware and new modeling software technology. This capability is predicted to lead to a better understanding of granular materials, contributing to many areas of research. However, these capabilities are only useful if they are shown to agree well with reality. Beginning at a simple level, a study was conducted to compare the simulation of primitive joints in the Chrono::Engine simulation package with the MSC/ADAMS commercial software package. This simple effort provides a measure for the accuracy with which the software models the interaction of a joint and one or two bodies. This allows more complex analyses to be carried out with knowledge of the software's accuracy at this level.
\end{abstract}

\section{Overview}

The capability to simulate billion-body models will, perhaps, soon be a reality. As computing power and simulation methods improve, the size of simulations can continue to grow [4]. Simulating many-body systems is important to the study of granular materials, since, in this field, it can be very difficult, if not impossible, to accurately measure the parameters of the particles [5]. Furthermore, simulation can be used to test new designs in low- or zero-gravity or other conditions that are infeasible to access or create in the real world [1].

While simulating granular materials clearly can offer many benefits over conducting physical experiments, this capability cannot be fully utilized until it is known to what extent simulation mirrors reality. Validation is essential to knowing whether simulations are modeling reality well, but to conduct a validation, measurements of reality are necessary. Since it is difficult to measure the parameters of granular materials, this study starts by comparing a simpler set of models involved in the multibody dynamic simulation of granular material: primitive joints.

This effort, while not directly addressing the issue of validating granular material simulations, provides assurance that the software behaves correctly for two bodies; it follows that the software should behave correctly for 20 bodies, 2,000 bodies, or 2,000,000 bodies. By comparing a more basic piece of the simulation software, one can check for inaccuracies while also providing a stepping stone for further validations.

The software in question in this effort, Chrono::Engine [7], [8], has been used to create and simulate large, many-body granular material models, such as the one pictured in Figure 1, which consists of 50,000 ellipsoidal particles [2]. MSC/ADAMS, a well-known and well-validated

The authors would like to thank the Wisconsin Space Grant Consortium Research Infrastructure Program for financial support. 
software package [3], provided proven values with which the Chrono::Engine results were compared. Primitive joint models were created in both software packages and direct measurements (position, velocity, acceleration, joint reaction force, and joint reaction torque) from the simulation of these models were examined.

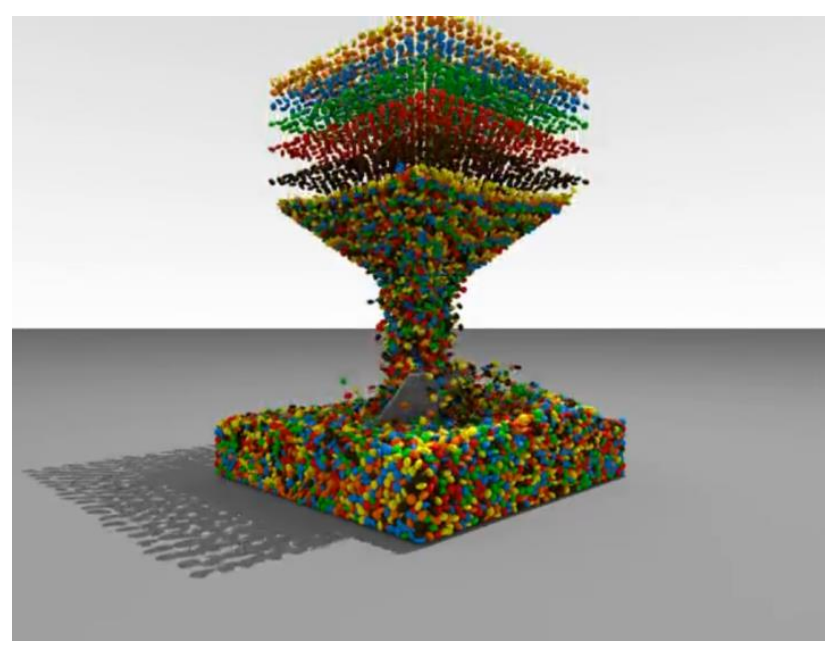

Figure 1: Granular simulation of 50,000 ellipsoidal particles, simulated using Chrono::Engine*

\section{Comparison}

Introduction. This study compared the simulation of primitive joints in Chrono::Engine and MSC/ADAMS 2010 (ADAMS). By considering the measured position, velocity, acceleration, joint reaction force, and joint reaction torque results from simple models in these two software packages, the project analyzed one basic piece of the Chrono::Engine software.

Methods. To carry out this comparison, a simple model was created for each of a list of primitive joints, shown in Table 1. Identical versions of each model were made in Chrono::Engine and ADAMS. Then the results generated by the simulation of this model in the two software packages were compared. The models each contain one primitive joint which connects a moving body to a fixed body (the ground). There are no applied forces in the models; the bodies are acted on only by gravity. Keeping the models simple allows the results to be more easily analyzed.

Models. The joints that were analyzed in this study are listed in Table 1, with data on the number of degrees of freedom allowed and removed by each joint. The "other" columns in the table refer to the angled directions of translation and axes of rotation. For example, for the angled translational joint, the "other" direction of translation refers to a path that is negative 45 degrees from the positive $\mathrm{x}$ axis. The orientation is explained further in the description of the translational joint models.

For the first three joints listed in Table 1 (the "pendulum joints": the revolute, Hooke, and spherical joints), the models created for the comparison, one of which is shown in Figure 2, each consist of a simple pendulum: one link that is fixed at one end to the ground, allowing the other end to move freely.

\footnotetext{
${ }^{*}$ A video rendering of this simulation can be viewed at http://sbel.wisc.edu/Animations/.
} 
Three models were created for each of the remaining three joints from Table 1 (the "slider joints": the translational, cylindrical, and planar joints) to consider three different joint orientations; examples of these models can be seen in Figure 7. In each model, a body is fixed to the ground through its center of mass by the joint in question. In the first model for each joint, the joint is oriented vertically, offering no resistance to the body's downward motion due to gravity. In the second model, the joint is oriented horizontally; thus, the joint holds the body in place and prevents it from falling. In the third model, the joint is placed at an angle of negative 45 degrees from the positive $\mathrm{x}$ axis (horizontal). Thus, the joint allows the body to slide down the incline in the positive $\mathrm{x}$, negative $\mathrm{y}$ direction.

Table 1: List of joints considered in this validation and the degrees of freedom (DOF) allowed and removed by each joint. The "other" columns refer to translational paths and rotational axes that are not oriented along the $x, y$, or $z$ axes.

\begin{tabular}{|c|c|c|c|c|c|c|c|c|c|c|c|}
\hline \multirow[t]{2}{*}{ Joint } & \multicolumn{4}{|c|}{$\begin{array}{c}\text { Translations } \\
\text { Allowed }\end{array}$} & \multirow{2}{*}{$\begin{array}{c}\text { Translational } \\
\text { DOF }\end{array}$} & \multicolumn{4}{|c|}{$\begin{array}{l}\text { Rotations } \\
\text { Allowed }\end{array}$} & \multirow{2}{*}{$\begin{array}{l}\text { Rotational } \\
\text { DOF }\end{array}$} & \multirow{2}{*}{$\begin{array}{c}\text { DOF } \\
\text { Removed } \\
\text { by Joint }\end{array}$} \\
\hline & $\mathbf{x}$ & $y$ & $\mathbf{z}$ & Other & & $\mathbf{x}$ & $\mathbf{y}$ & $\mathbf{z}$ & Other & & \\
\hline Revolute Joint & - & - & - & - & 0 & - & - & $\mathrm{x}$ & - & 1 & 5 \\
\hline Hooke Joint & - & - & - & - & 0 & $x$ & - & $\mathrm{x}$ & - & 2 & 4 \\
\hline Spherical Joint & - & - & - & - & 0 & $\mathrm{x}$ & $\mathrm{x}$ & $\mathrm{x}$ & - & 3 & 3 \\
\hline \multicolumn{12}{|c|}{ Translational Joint } \\
\hline Vertical & $\mathrm{x}$ & $x$ & - & - & 1 & - & - & - & - & 0 & 5 \\
\hline Horizontal & $\mathrm{x}$ & - & - & - & 1 & - & - & - & - & 0 & 5 \\
\hline Angled & - & - & - & $\mathrm{x}$ & 1 & - & - & - & - & 0 & 5 \\
\hline \multicolumn{12}{|l|}{ Cylindrical Joint } \\
\hline Vertical & - & $x$ & - & - & 1 & - & $\mathrm{x}$ & - & - & 1 & 4 \\
\hline Horizontal & $\mathrm{x}$ & - & - & - & 1 & $x$ & - & - & - & 1 & 4 \\
\hline Angled & - & - & - & $x$ & 1 & - & - & - & $x$ & 1 & 4 \\
\hline \multicolumn{12}{|l|}{ Planar Joint } \\
\hline Vertical & $\mathrm{x}$ & $x$ & - & - & 2 & - & - & $\mathrm{x}$ & - & 1 & 3 \\
\hline Horizontal & $\mathrm{x}$ & - & $x$ & - & 2 & - & $x$ & - & - & 1 & 3 \\
\hline Angled & -1 & - & $\mathrm{x}$ & $x$ & 2 & - & - & - & $\mathrm{x}$ & 1 & 3 \\
\hline
\end{tabular}

Results. The full results from the comparison can be found in [6]. The results of the first joint from each group are presented in the following two sections.

Revolute Joint. In the Chrono::Engine and ADAMS simulations, a simple pendulum featuring a revolute joint was created. The pendulum, which consists of a link with a mass of $1 \mathrm{~kg}$, is pinned, on one end of the link, at the origin. There are no forces acting on the pendulum besides gravity, which acts in the negative y (downward) direction. The initial position of the link is horizontal, along the positive $\mathrm{x}$ axis. The model in ADAMS is shown in its initial position in Figure 2. The pendulum begins at rest. The model is oriented in the $x-y$ plane; therefore, the results in the $\mathrm{z}$ direction are all zero. 


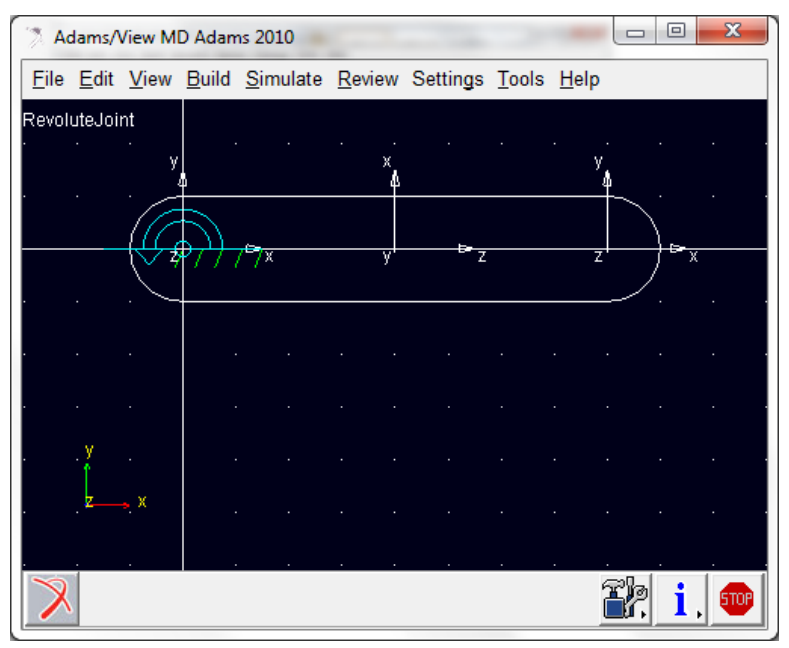

Figure 2: Screenshot of revolute joint pendulum model in ADAMS

The position and velocity results from Chrono::Engine (Chrono) and ADAMS (Adams) (see Figure 3 and Figure 4) show good agreement with only small, though apparently increasing, errors in the $\mathrm{x}$ and $\mathrm{y}$ directions. The errors in the average position magnitude and average velocity magnitude have values of 0.001065 and 0.035105 , which are $0.053 \%$ and $1.012 \%$ of the ADAMS results, respectively.
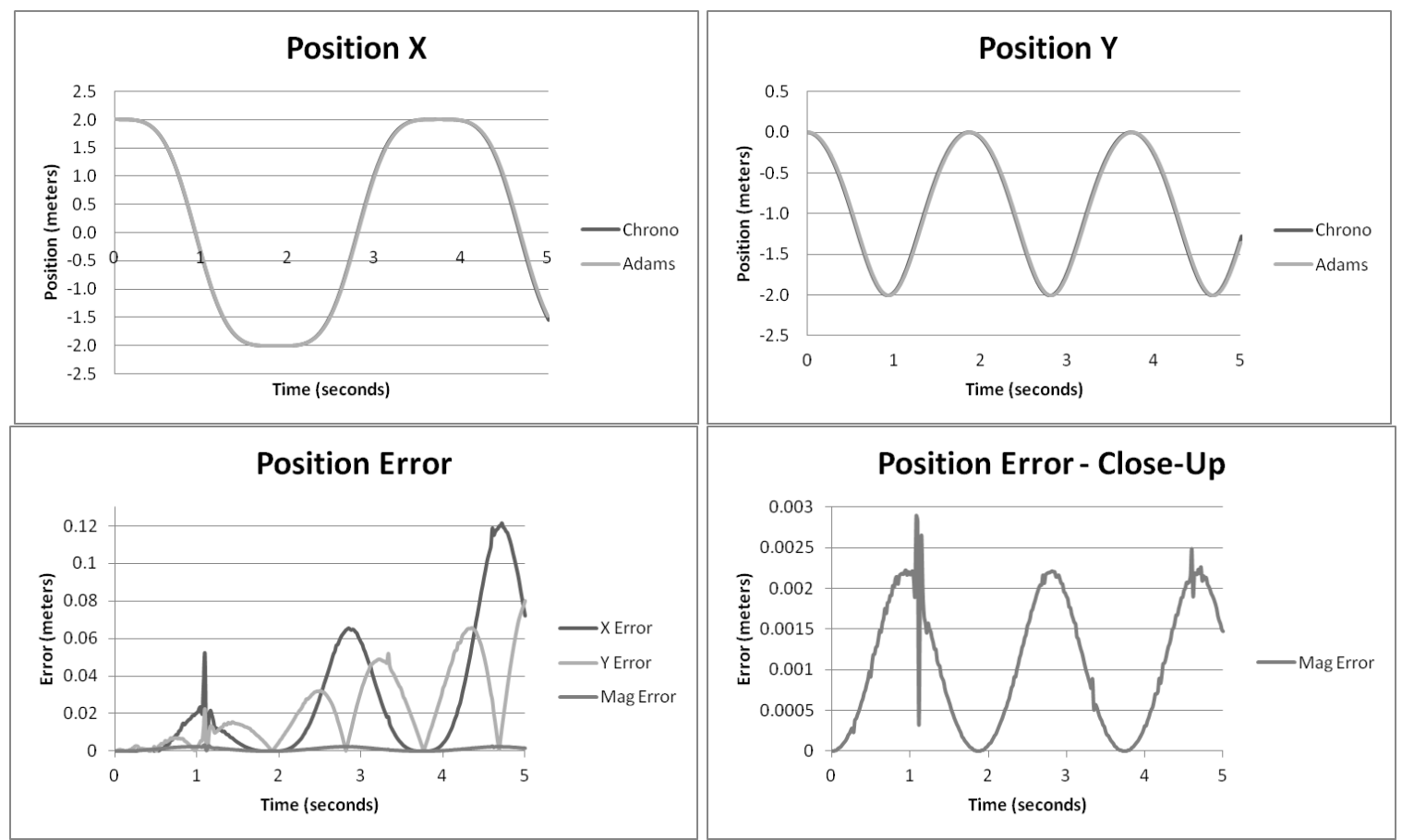

Figure 3: Position results in the $\mathrm{x}$ and $\mathrm{y}$ directions and error magnitude for the revolute joint models 

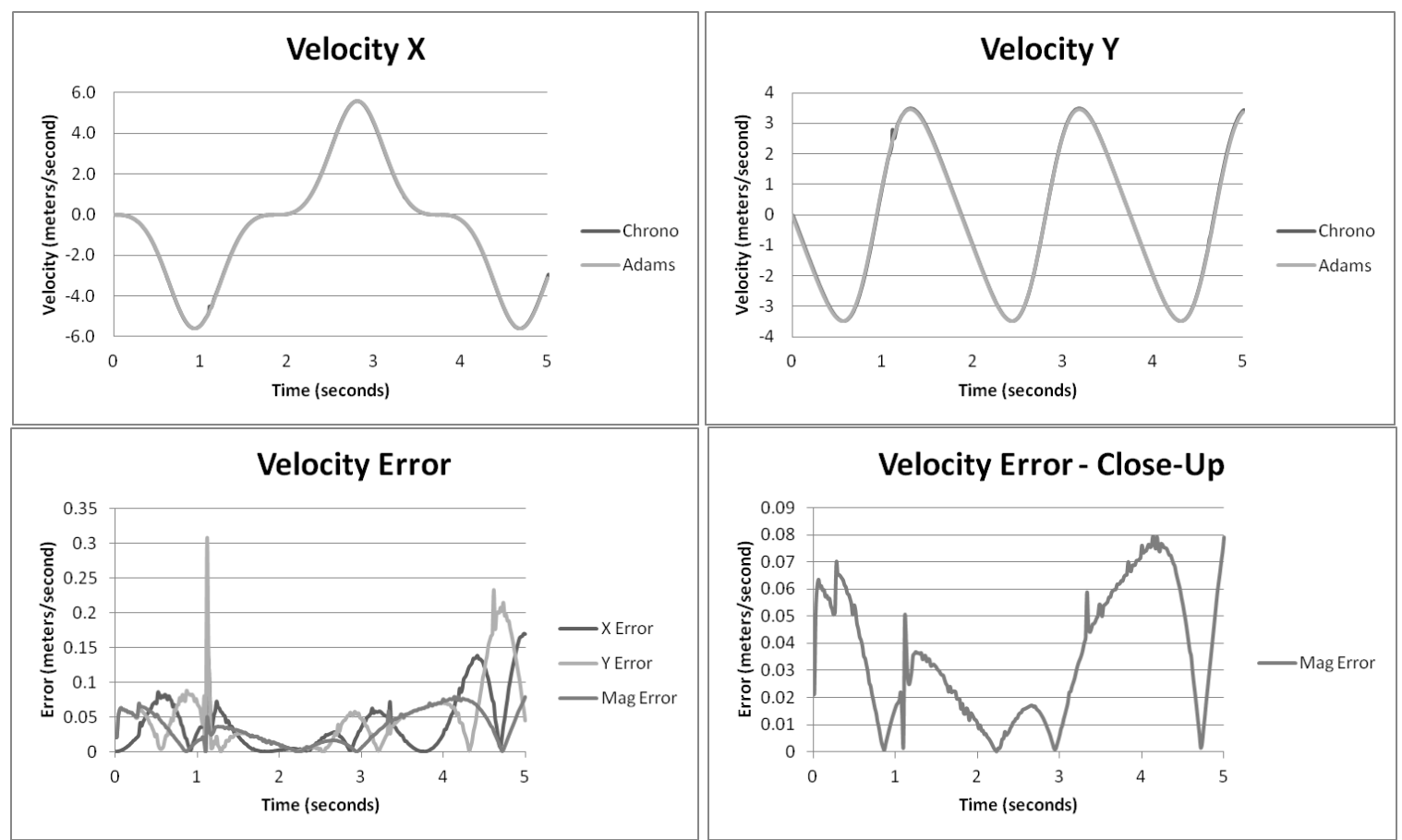

Figure 4: Velocity results in the $\mathrm{x}$ and $\mathrm{y}$ directions and error magnitude for the revolute joint models

The acceleration results, shown in Figure 5, have more discrepancies, the most significant of which is the spike that occurred in the Chrono::Engine simulation at a time of just past 1 second; this is obvious in the $\mathrm{x}, \mathrm{y}$, and error acceleration plots, and can also be seen as a small inconsistency in the $\mathrm{x}, \mathrm{y}$, and error velocity plots in Figure 4. The error in the average acceleration magnitude has a value of 0.485406 , which is $4.437 \%$ of the ADAMS results.

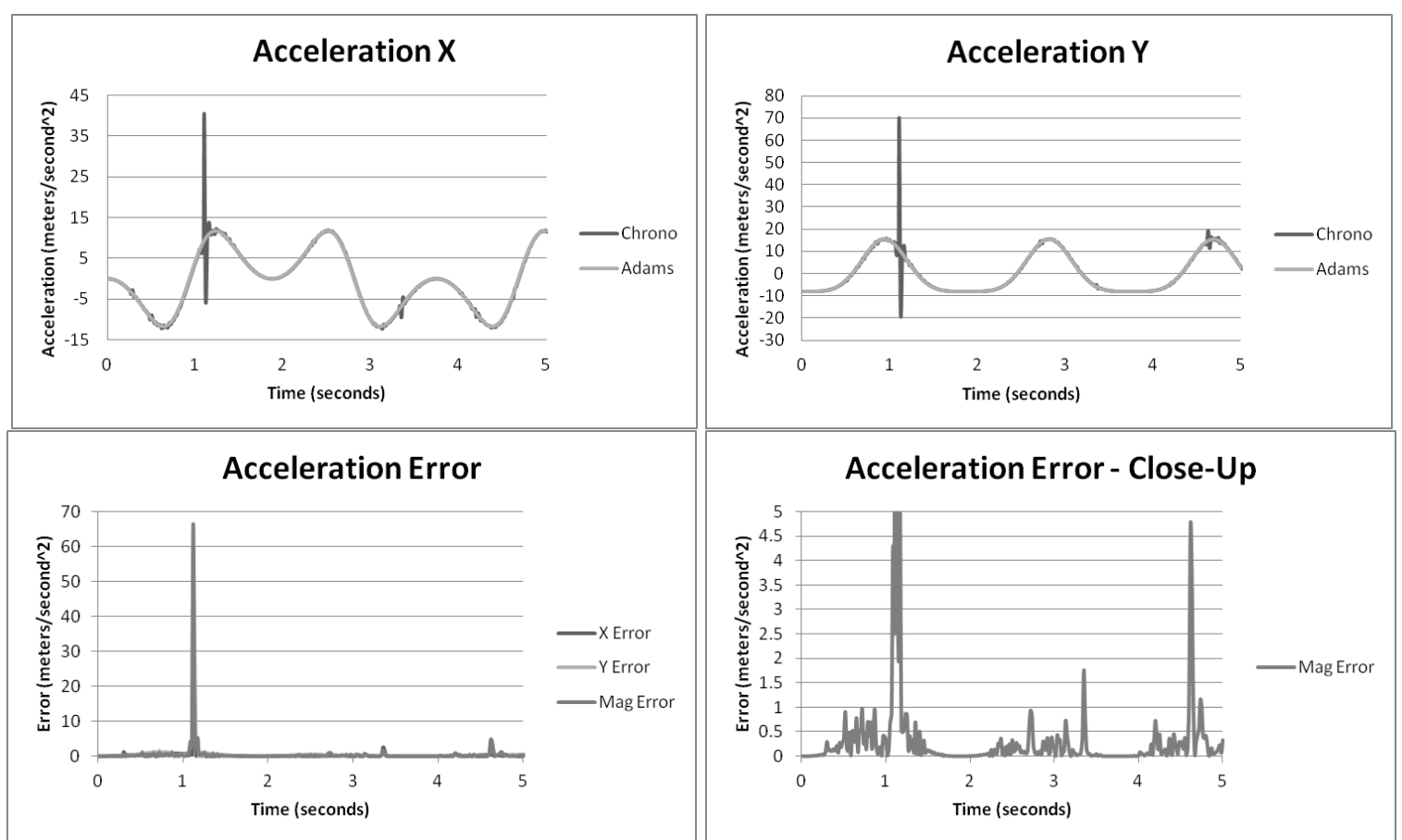

Figure 5: Acceleration results in the $\mathrm{x}$ and $\mathrm{y}$ directions and error magnitude for the revolute joint models 
The joint reaction forces are presented in Figure 6. These plots show poor agreement between the two software packages for the forces in the $\mathrm{x}$ and $\mathrm{y}$ directions; the shapes of the force plots are different, as are the values of the force. However, as is shown by the fact that the magnitudes of the forces are very similar, the difference in the $\mathrm{x}$ and $\mathrm{y}$ directions is mainly due only to a difference in the definitions of the reference frames. The error in the average reaction force magnitudes has a value of 0.6278 , which is $4.928 \%$ of the ADAMS results.

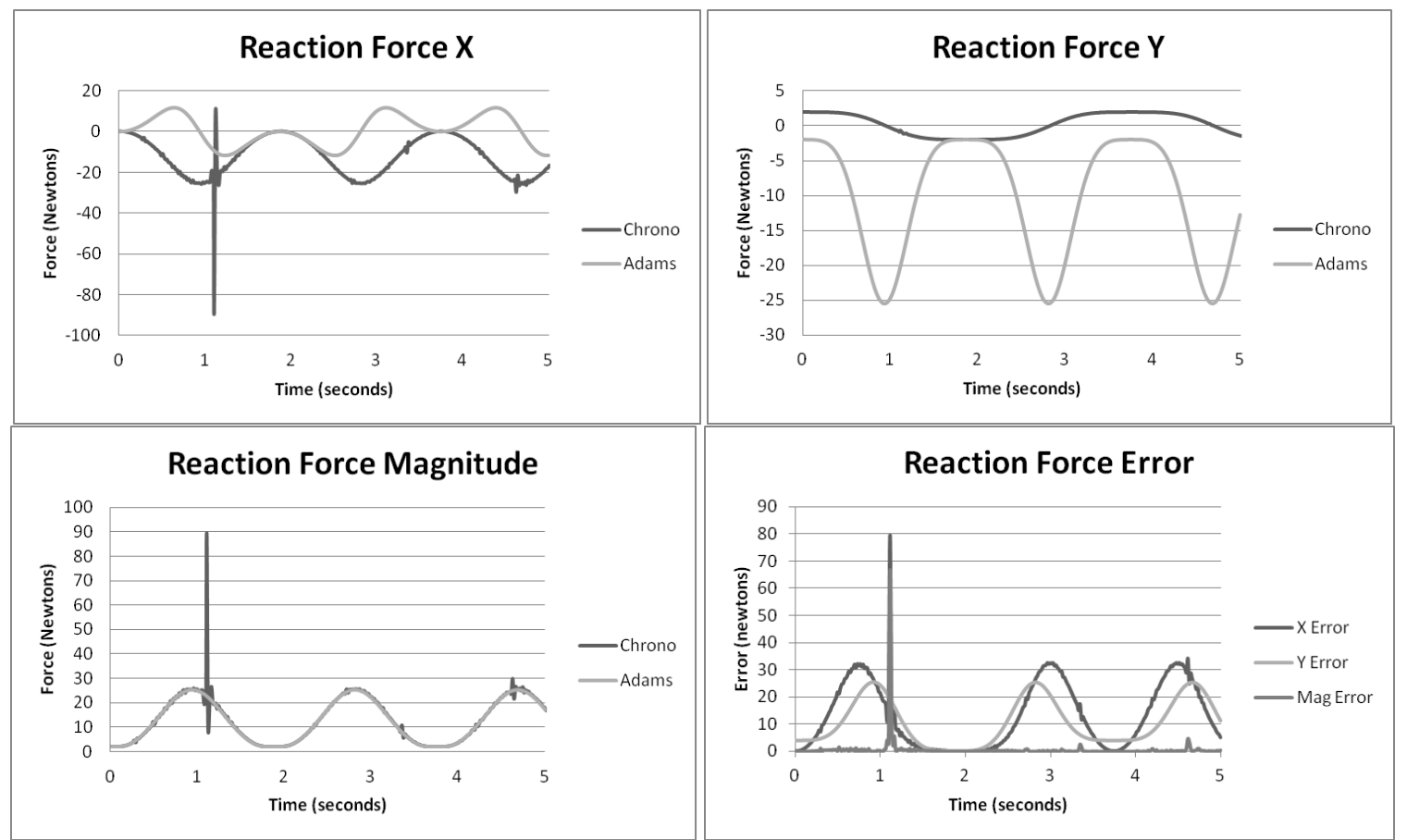

Figure 6: Joint reaction force results in the $\mathrm{x}$ and $\mathrm{y}$ directions, magnitude, and error magnitude for the revolute joint models

Translational Joint. For the translational joint, three models were created in each Chrono::Engine and ADAMS. Each model consists of a body with a mass of $1 \mathrm{~kg}$. The body is fixed, though its center of mass, to the ground, at the origin, by a translational joint. In the first model, the joint is oriented vertically; for the second it is horizontal, and for the third it is oriented at a 45 degree angle down (clockwise) from the positive $\mathrm{x}$ axis (horizontal). The ADAMS models, in their initial positions, are shown in Figure 7. There are no applied forces; the body in each model is acted on only by the joint and gravity. The initial position of the body in each model is at the origin, and the body starts from rest. The models are located in the $x-y$ plane; therefore, the results in the $\mathrm{z}$ direction are omitted. 

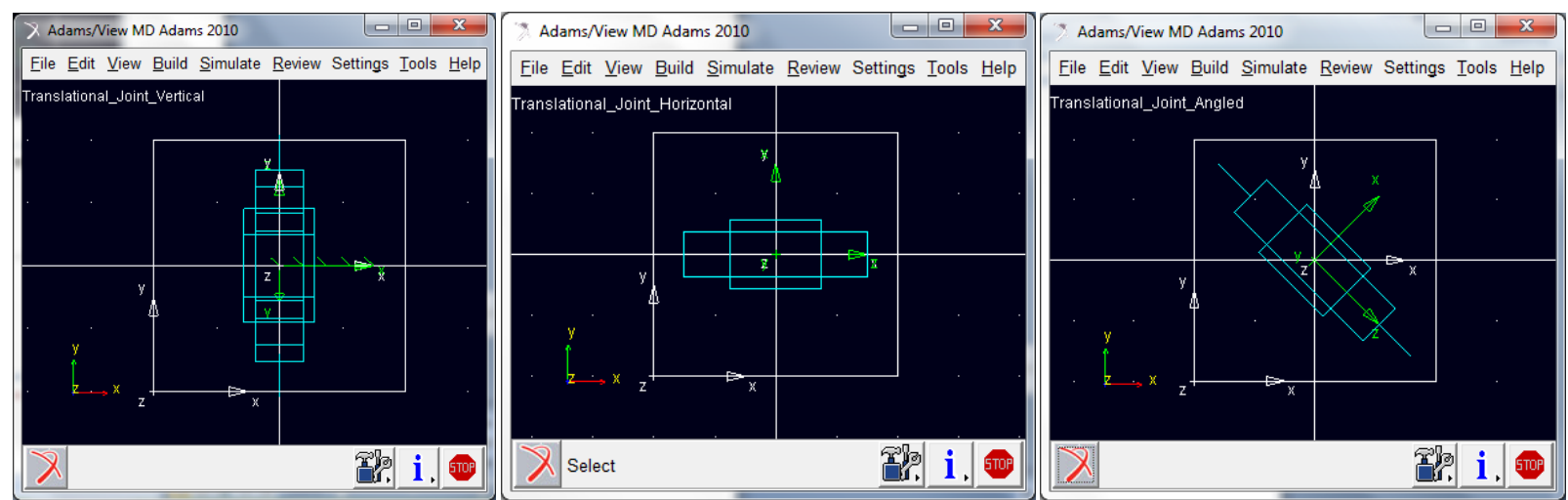

Figure 7: Screenshots of translational joint models in ADAMS; the joints are oriented, from left to right, vertically, horizontally, and at an angle

The position, velocity, and acceleration results show good agreement between Chrono::Engine and ADAMS; a selection of this data is shown in Figure 8 for the vertically-oriented joint and in Figure 9 for the angled joint. A summary of the error data for the three models is also shown in Table 2.

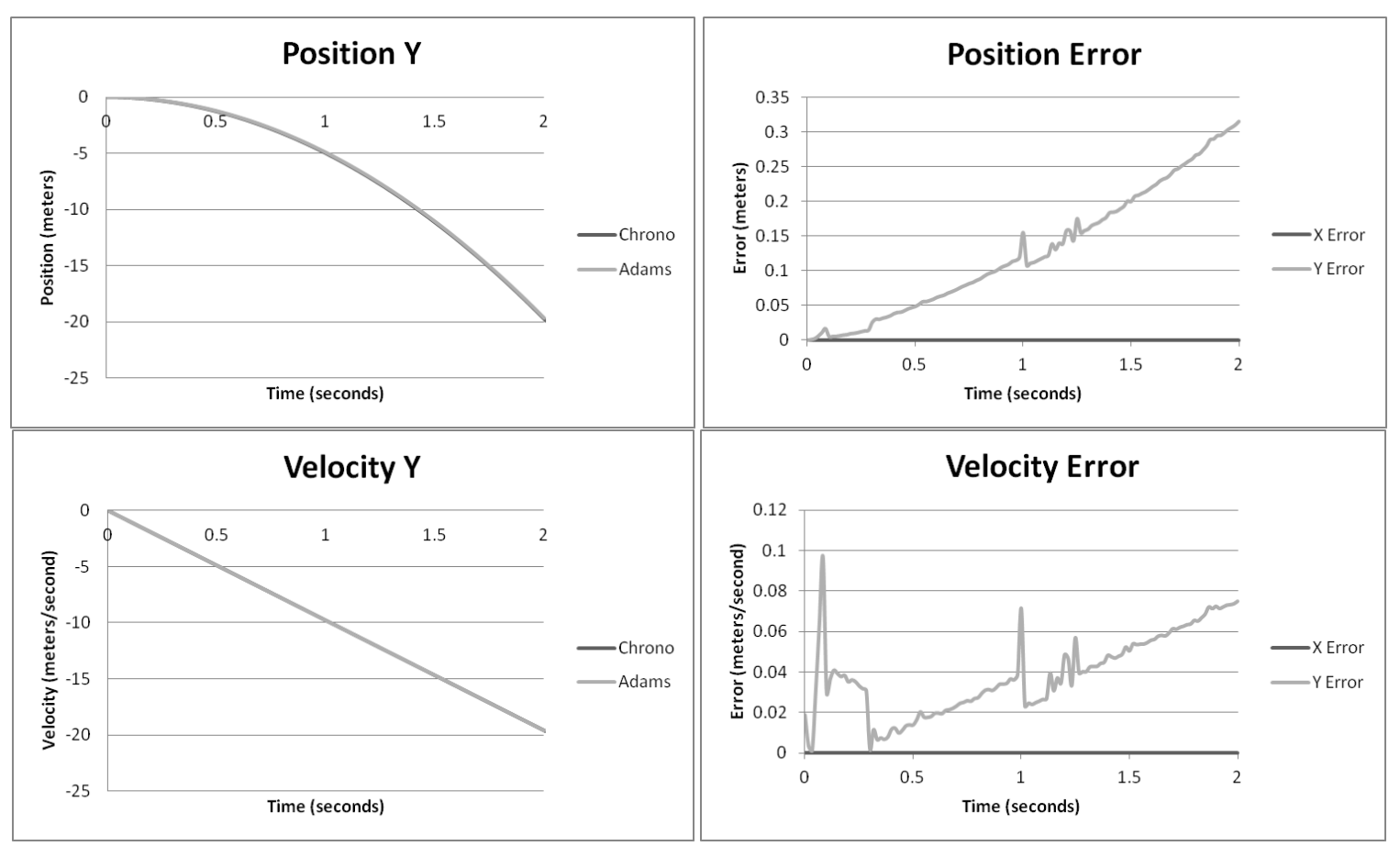

Figure 8: Position and velocity results in the y direction and error magnitudes for the vertically-oriented translational joint models 

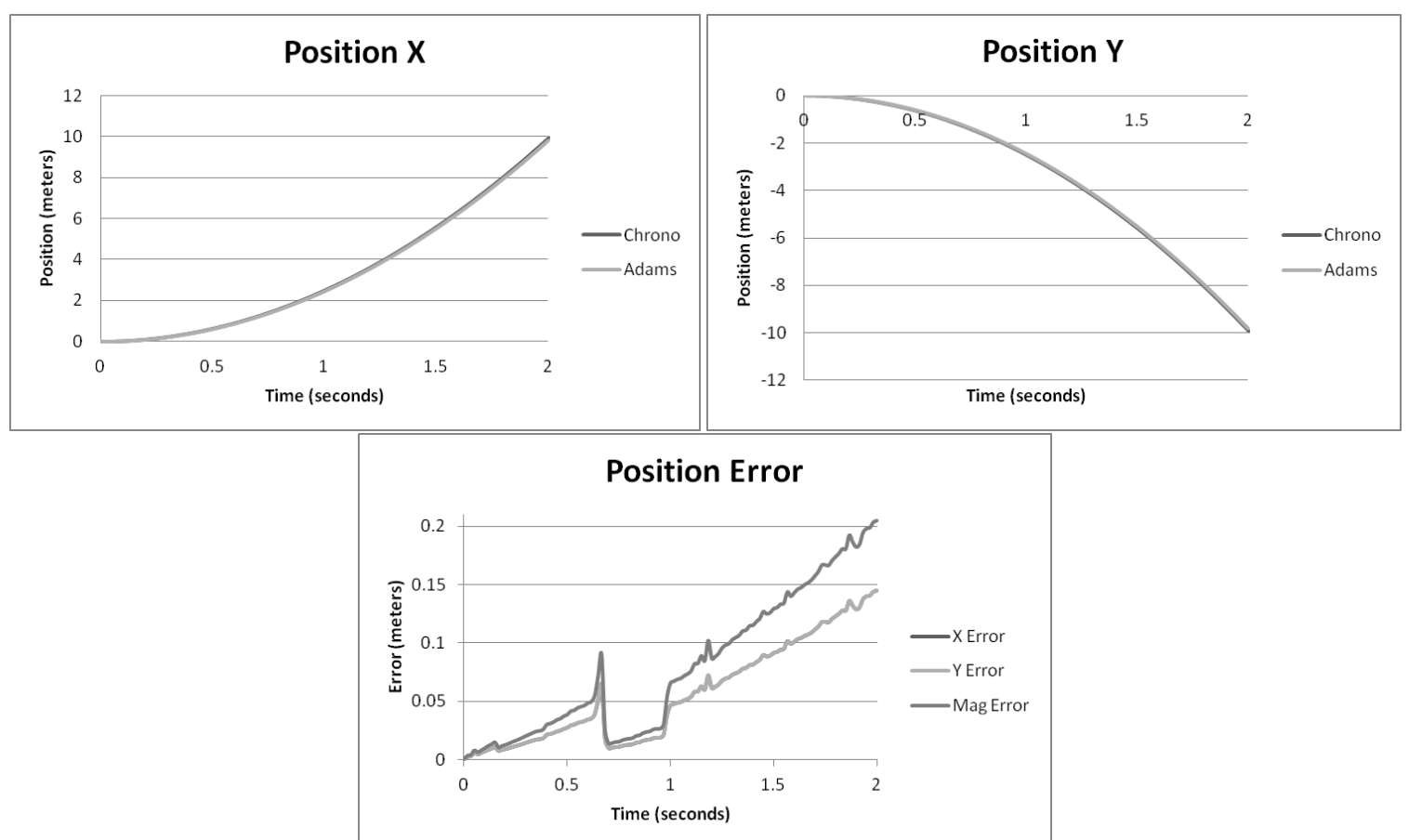

Figure 9: Position results in the $\mathrm{x}$ and $\mathrm{y}$ directions and error magnitude for the angled translational joint models

Table 2: Average error data for translational joint models (horizontal data is omitted since the differences between the magnitudes were either zero or very small)

\begin{tabular}{|l|l|l|c|l|}
\hline Error Data for Vertical and Angled Translational Joint Models \\
\hline & \multicolumn{2}{|c|}{ Vertical: } & \multicolumn{2}{c|}{ Angled: } \\
\hline Error in: & Error & $\%$ of ADAMS & Error & $\%$ of ADAMS \\
\hline Avg Position Mag & 0.129174761 & $1.9676 \%$ & 0.079135785 & $1.7047 \%$ \\
\hline Avg Velocity Mag & 0.038781457 & $0.3955 \%$ & 0.027974969 & $0.4034 \%$ \\
\hline Avg Acceleration Mag & $1.2562 \mathrm{E}-05$ & $0.0001 \%$ & $5.82645 \mathrm{E}-06$ & $0.0001 \%$ \\
\hline Avg Reaction Force Mag & $5.84522 \mathrm{E}-07$ & - & 0.000463661 & $0.0067 \%$ \\
\hline
\end{tabular}

The joint reaction force results (see Table 2 and Figure 10Figure 11Figure 12) show some discrepancies between the two software packages. For the vertically-oriented joint models, the difference in the force results between the two software packages is quite small (at most, approximately $6 \mathrm{E}-7 \mathrm{~N}$ ), as Figure 10 shows. For the horizontally-oriented joint, the reaction force in the y direction is negative in Chrono::Engine and positive in ADAMS, as can be seen in Figure 11. This is only a difference in definition, as the forces calculated have the same magnitude. A difference in definition can also be seen in the results from the angled translational joint models (see Figure 12). The forces in the $\mathrm{x}$ and $\mathrm{y}$ directions agree poorly for this model; however, the mean magnitudes of the forces are the same, providing evidence that the Chrono::Engine force calculations are indeed accurate. The Chrono::Engine results do show some discrepancies from the ADAMS results for the angled translational joint model; the force magnitude deviates from the mean value, with the deviations appearing to increase with time. 


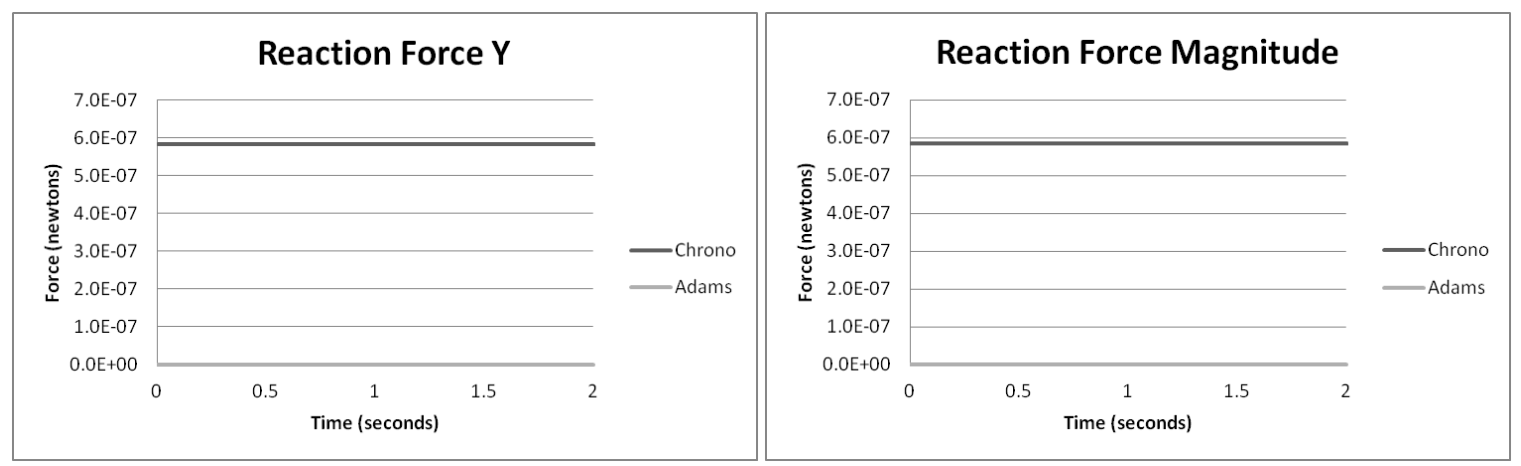

Figure 10: Joint reaction force data in the y direction and magnitude for the vertically-oriented translational joint models
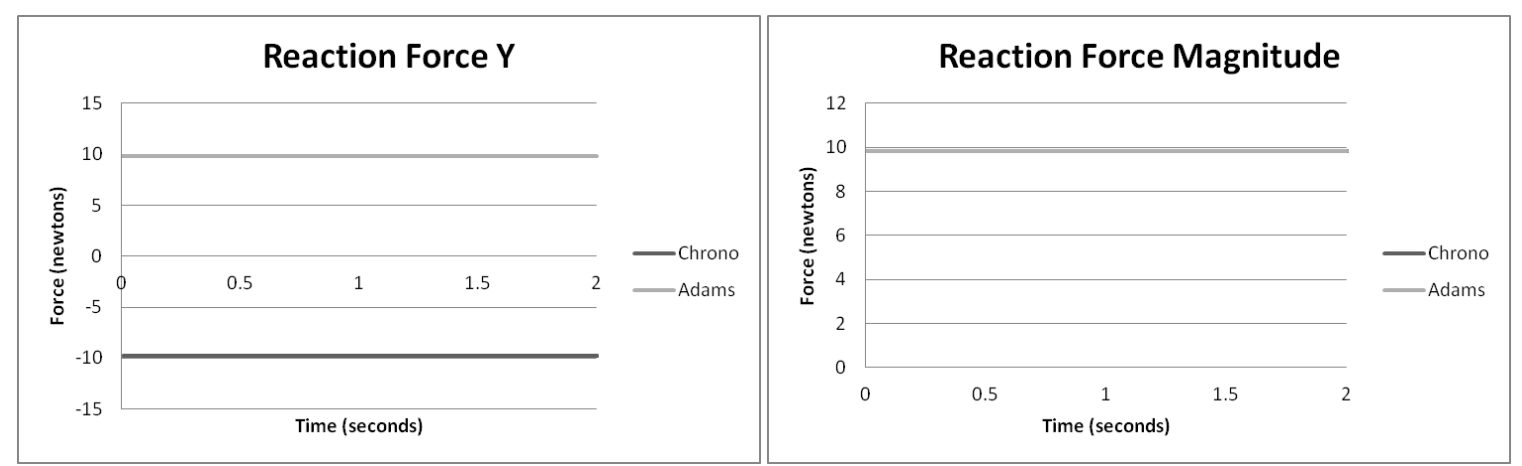

Figure 11: Joint reaction force data in the y direction and magnitude for the horizontally-oriented translational joint models

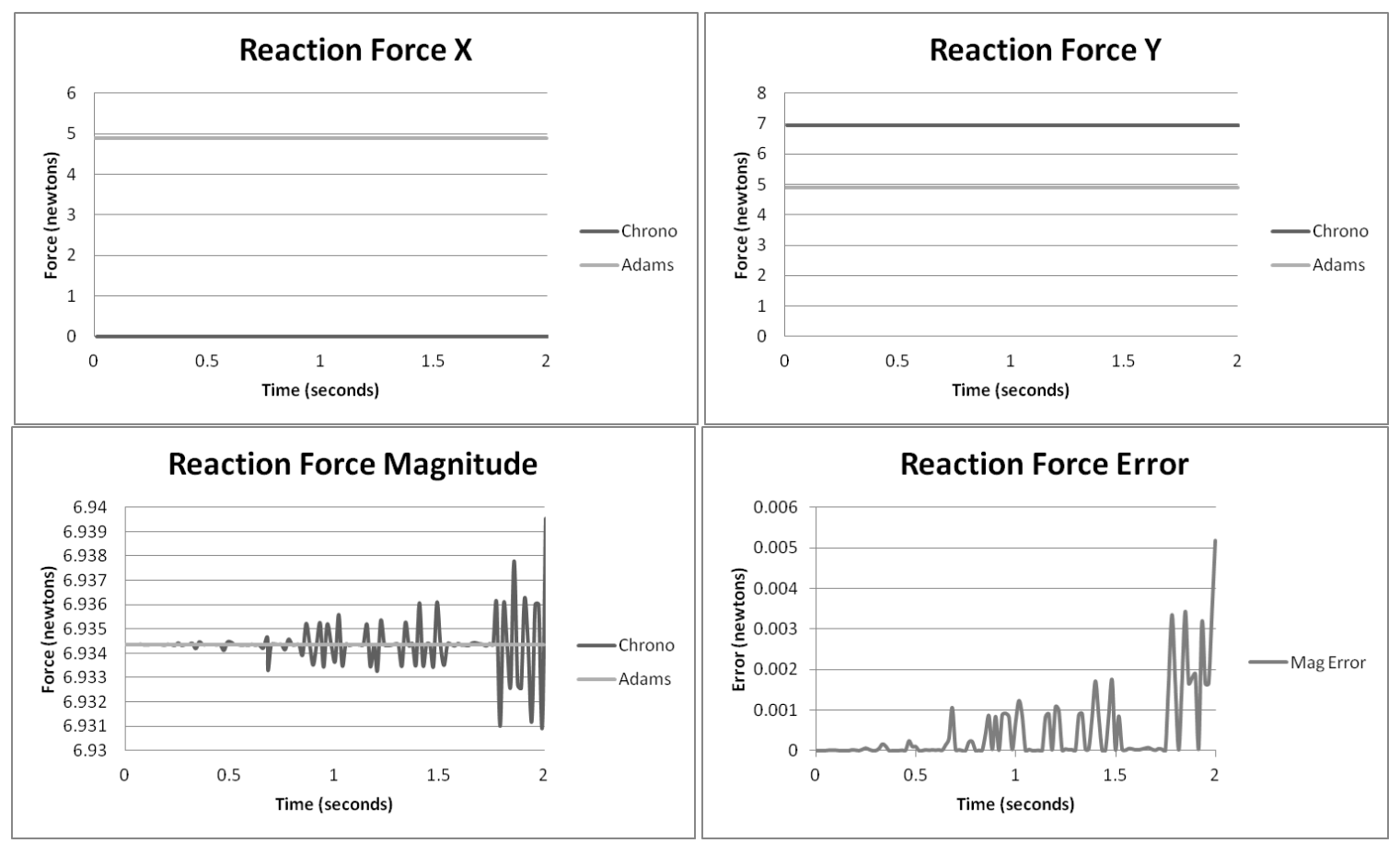

Figure 12: Joint reaction force data in $\mathrm{x}$ and $\mathrm{y}$ directions, magnitude, and error magnitude for angled translational joint models 


\section{Future Plans and Conclusion}

Overall, this effort was a straightforward means of $(i)$ providing assurance that Chrono::Engine simulations are accurate, and (ii) working towards validating the software's many-body granular material simulations.

This effort provides evidence that Chrono::Engine generates the same or similar position, velocity, acceleration, and joint reaction force results for the primitive joints considered. The study also highlighted some areas for further investigation, specifically, the spikes in the acceleration and joint reaction forces for the revolute joint models and the discrepancies in the joint reaction forces for the angled translational joint models. These topics will be addressed more fully in future work.

To continue the effort of providing a measure of accuracy for Chrono::Engine, future work will also include validations of the software's granular material simulations against experimental results. Comparing to ADAMS simulations, as in the current effort, is infeasible for use in directly validating the simulation of granular materials due to the program's limited efficiency for even very small systems. Therefore, future direct validations of granular material simulations will involve designing and creating physical models from which experimental data (such as mass flow rate) will be gathered.

The direct validation process, outlined in the previous paragraph, is obviously not as straightforward as creating models in ADAMS. This highlights the benefit of the current work in that it provided a simple method for lending greater confidence in Chrono::Engine results, bringing us one step closer to accurate, billion-body simulations.

\section{References}

[1] Mazhar, H., Quadrelli, M., Negrut, D., Using a Granular Dynamics Code to Investigate the Performance of a Helical Anchoring System Design: Technical Report TR-2011-03, 2011, Simulation-Based Engineering Lab, University of Wisconsin - Madison.

[2] Mazhar, H., Granular Simulation of 50,000 Ellipsoids, http://sbel.wisc.edu/Animations/.

[3] MSC.Software, ADAMS Standard Documentation and Help, 2010, MSC Software Corporation, ADAMS 2010.

[4] Negrut, D., A. Tasora, and M. Anitescu, Large-scale parallel multibody dynamics with frictional contact on the GPU, in ASME 2008 Dynamic Systems and Control Conference, E. Misawa, Editor 2008, ASME: Ann Arbor, MI.

[5] Pöschel, T. and T. Schwager, Computational granular dynamics: models and algorithms. 2005: Springer.

[6] Shotwell, R., A Comparison of Chrono::Engine's Primitive Joints against ADAMS Results: Technical Report TR-2012-01, 2012, Simulation-Based Engineering Lab, University of Wisconsin - Madison.

[7] Tasora, A. Chrono::Engine, 2012, Available from: http://www.deltaknowledge.com/chronoengine/.

[8] Tasora, A., M. Silvestri, and P. Righettini, Architecture of the Chrono::Engine physics simulation middleware, in ECCOMAS Multibody Dynamics Conference 2007: Milano. p. 1-8. 